\title{
Evaluating Sarcopenia by Using the Bioelectrical Impedance Analysis in Patients with Acute Myeloid Leukemia After Chemotherapy
}

\author{
Zheng Qin', Kai Lu², Tiantian Jiang ${ }^{3}$, Mei Wang ${ }^{3}$, Yue Weng', Xiaoqiong Tang ${ }^{3}$, Yu Zhao' \\ 'Department of Vascular Surgery, The First Affiliated Hospital of Chongqing Medical University, Chongqing, 4000I6, People's Republic of China; \\ ${ }^{2}$ Department of Cardiology, The First Affiliated Hospital of Chongqing Medical University, Chongqing, 4000I6, People's Republic of China; \\ ${ }^{3}$ Department of hematopathology, The First Affiliated Hospital of Chongqing Medical University, Chongqing, 4000I6, People's Republic of China
}

Correspondence: Xiaoqiong Tang, Department of Hematopathology, The First Affiliated Hospital of Chongqing Medical University, I Youyi Road, Yuzhong, Chongqing, 400016, People's Republic of China, Email tlscqmu@I26.com; Yu Zhao, Department of Vascular Surgery, The First Affiliated Hospital of Chongqing Medical University, I Youyi Road, Yuzhong, Chongqing, 4000I6, People's Republic of China,

Email zhaoyucqmu@126.com

Background: We aimed to explore the potential association of body composition parameters measured by bioelectrical impedance analysis (BIA) with the incidence of sarcopenia in patients with acute myeloid leukemia (AML) (non-M3) after chemotherapy.

Patients and Methods: This was a single-center observational study. Sixty-nine patients with newly diagnosed AML underwent BIA at the time of initial diagnosis and after completion of three chemotherapy sessions. Pre- and post-chemotherapy BIA parameters were compared. Sarcopenia was defined as low skeletal muscle mass plus low muscle strength according to the Asian Working Group for Sarcopenia (AWGS). Association of sarcopenia with mid-arm muscle circumference (MAMC) and intracellular water (ICW) was assessed by multivariate logistic regression.

Results: There was a significant increase in the prevalence of sarcopenia after chemotherapy $(39.1 \%$ vs $14.5 \%, P<0.001)$. Skeletal muscle mass (SMM), fat-free mass (FFM), and soft lean mass (SLM) showed a significant decrease after chemotherapy $(P<0.05)$. MAMC, ICW, and total body water (TBW) significantly decreased after chemotherapy $(P<0.05)$. BIA indices including appendicular skeletal muscle mass (ASM) $(r=0.889, P<0.001)$, ICW $(r=0.869, P<0.001)$, MAMC $(r=0.849, P<0.001)$ showed a positive correlation with SMI. Moreover, ASM $(r=-0.453 P=0.001)$, ICW $(r=-0.322, P<0.05)$, and MAMC $(r=-0.352, P<0.05)$ showed a negative correlation with sarcopenia. On multivariate logistic regression analysis, increased ICW was associated with decreased risk of sarcopenia [odds ratio (OR): $0.50 ; 95 \%$ confidence interval (CI) $0.30-0.82$ ]. Each additional unit of MAMC after chemotherapy was associated with $71 \%$ lower risk of sarcopenia (OR: 0.29 ; 95\% CI $0.13-0.66$ ).

Conclusion: The incidence of sarcopenia was associated with chemotherapy of patients with AML (non-M3) as reflected by body composition changes.

Keywords: acute myeloid leukemia, sarcopenia, bioelectrical impedance analysis, mid-arm muscle circumference

\section{Introduction}

Acute myeloid leukemia (AML) is one of the most common forms of leukemia in adults. ${ }^{1}$ In the absence of treatment, death usually occurs within months of diagnosis secondary to infection or bleeding. ${ }^{2}$ Chemotherapy is the main treatment modality for AML. However, the side effects of chemotherapy, including body composition disorders, dramatically decrease the quality of life and lead to poor prognosis. ${ }^{3}$ Therefore, prevention and early detection of post-chemotherapy changes in body composition is a key imperative for AML patients.

Muscle wasting is a critical component of the change in body composition. Sarcopenia is characterized by severe loss of muscle mass and decline in muscle function. ${ }^{4}$ The incidence of sarcopenia in elderly patients with hematologic malignancies, especially in non-Hodgkin's lymphoma (NHL), is higher than general elderly population. ${ }^{5}$ Several studies have corroborated the poor prognostic impact of sarcopenia and body composition changes in patients with various solid cancers. ${ }^{6-8}$ Sun et al 
reported that progress of sarcopenia in patients with AML is associated with adverse outcomes, and the overall survival (OS) of patients with sarcopenia was shorter than non-sarcopenia patients. ${ }^{9}$

Bioelectrical impedance analysis (BIA) has been widely used to evaluate body composition in various clinical fields. ${ }^{10}$ Devices for BIA have a high accuracy and efficiency for evaluation of body composition at a relatively low cost. ${ }^{11}$ In the contemporary literature, changes in body composition and occurrence of sarcopenia in AML (non-M3) patients after repeated sessions of chemotherapy are not well characterized.

The purpose of this study was to investigate the post-chemotherapy changes in body composition indicators of AML patients (non-M3), as measured by BIA, and to assess their potential association with the incidence of sarcopenia.

\section{Patients and Methods}

\section{Study Design and Population}

This was a single-center, observational study conducted from July 2020 to July 2021 in the Department of Hematology at the First Affiliated Hospital of Chongqing Medical University. During the study reference period (Figure 1) a total of 105 patients were hospitalized due to newly diagnosed AML.

The inclusion criteria for this study were 1) adult patients (age $\geq 18$ years) with diagnosis of AML confirmed by pathological examination or MICM classification and 2) indications for chemotherapy evaluated by the hematologist. The exclusion criteria were 1) patients with acute promyelocytic leukemia; 2) patients with liver and/or kidney dysfunction or other contraindications for chemotherapy, and those who still could not tolerate chemotherapy after active treatment for the above contraindications; 3) pregnant women; 4) any other reason that the investigator considered inappropriate to participate in this study. Finally, 69 initial diagnosis AML (non-M3) patients who reached complete remission (CR) were recruited in this study, followed by two sessions of consolidation chemotherapy. All AML (non-M3) patients were given

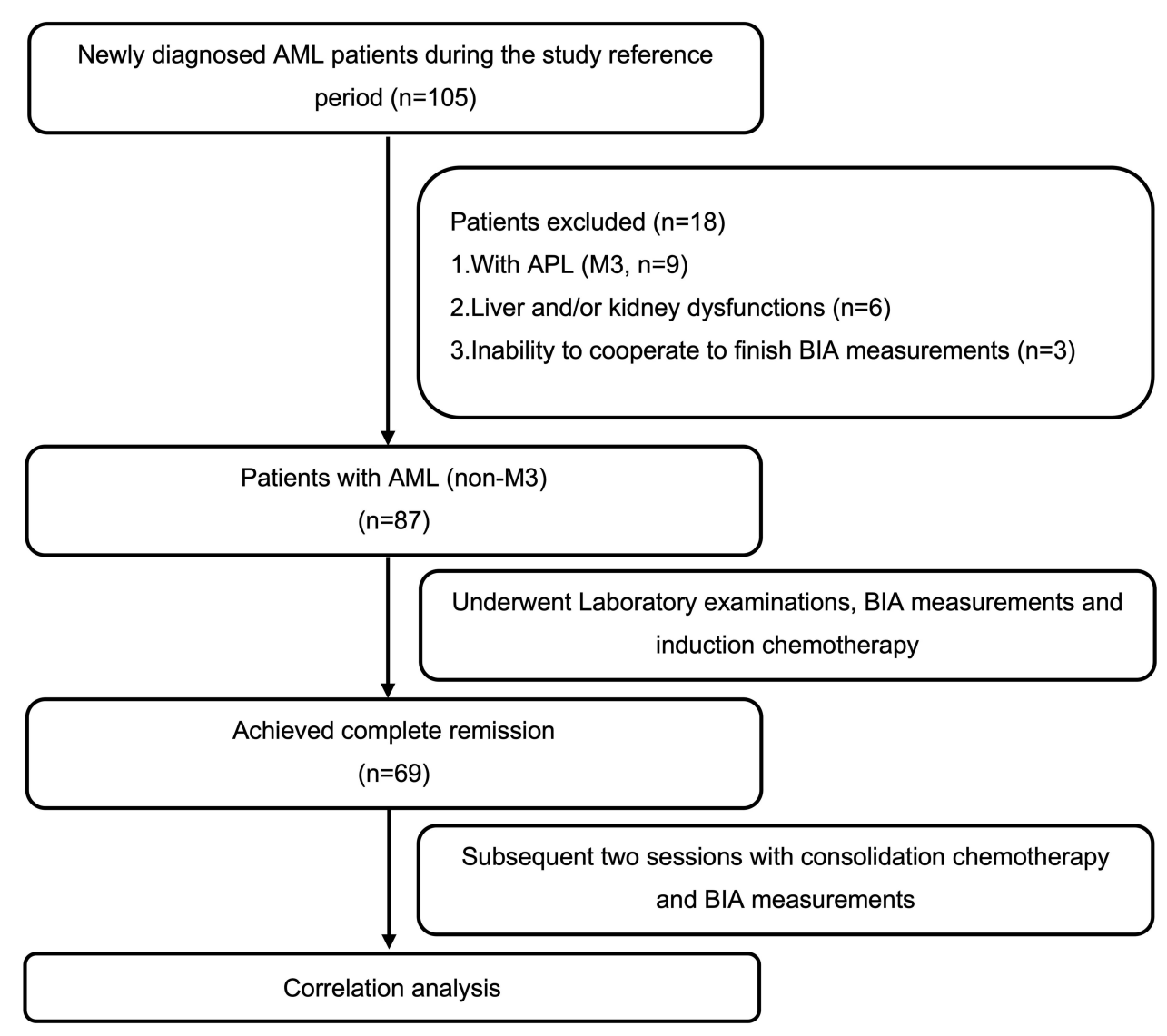

Figure I Schematic illustration of the study design and patient-selection criteria.

Abbreviations: AML, acute myeloid leukemia; APL, acute promyelocytic leukemia; BIA, bioelectric impedance analysis. 
the standard " $3+7$ " induction chemotherapy with idarubicin (IDA, $10 \mathrm{mg} / \mathrm{m}^{2} \cdot \mathrm{d}$ ) or daunorubicin (DNR, $60 \mathrm{mg} / \mathrm{m}^{2} \cdot \mathrm{d}$ ) plus cytarabine (Ara-c, $100-200 \mathrm{mg} / \mathrm{m}^{2} \cdot \mathrm{d}$ ). Consolidation chemotherapy consisted of high-dose cytarabine-based regimens (Ara-c, $1-3 \mathrm{~g} / \mathrm{m}^{2} \cdot \mathrm{q} 12$ ).

The purpose and content of this study were explained to all participants and their written informed consents were obtained prior to their enrolment. The study was conducted in accordance with the principles of the Declaration of Helsinki. Ethical approval was obtained from the First Affiliated Hospital of Chongqing Medical University (approval number 2020-589).

\section{Measurements and Definition of Body Composition Parameters}

Anthropometric variables include height $(\mathrm{cm})$, weight $(\mathrm{kg})$ and hand grip strength $(\mathrm{kg})$ were measured by doctors, following a standard protocol. ${ }^{12}$ Body mass index (BMI) was calculated as body weight in kilograms divided by height in meters squared $\left(\mathrm{kg} / \mathrm{m}^{2}\right)$. Routine laboratory tests were performed in the Department of Laboratory at the First Affiliated Hospital of Chongqing Medical University. All measurements and tests were undertaken at the time of initial diagnosis of AML and after completion of three chemotherapy sessions. All participants were subjected to BIA measurements by Direct Segmental Multi-Frequency Bioelectrical Impedance Analyzer (DSM-BIA, Inbody S10, Korea). After admission to the hospital, BIA measurements were performed prior to initiation of any fluid therapy following the manufacturer's instructions. All participants were instructed not to consume any food or drink and to avoid strenuous activity within 2 hours before the measurements.

All BIA parameters were obtained using a standard montage of outer and inner electrodes on the right hand and foot while patients lay down with legs apart. Body composition indicators including appendicular skeletal muscle mass (ASM), skeletal muscle mass (SMM), soft lean mass (SLM), intracellular water (ICW), total body water (TBW), armmuscle circumference (MAMC), visceral fat area (VFA); fat mass (FM) and protein content were measured and recorded. Sarcopenia was defined as low muscle mass plus low muscle strength according to the criteria of Asia Working Group for Sarcopenia (AWGS). ${ }^{13}$ Low muscle mass was defined as ASM corrected by height squared (appendicular skeletal muscle mass index, SMI) $\left(<5.7 \mathrm{~kg} / \mathrm{m}^{2}\right.$ in women and $<7.0 \mathrm{~kg} / \mathrm{m}^{2}$ in men). Low muscle strength was determined by low hand grip strength (HGS) $(<18 \mathrm{~kg}$ in women and $<28 \mathrm{~kg}$ in men).

\section{Statistical Analysis}

Continuous variables were presented as median with upper and lower quartiles, the interquartile range, or mean \pm stand deviation. Between-group differences with respect to normally distributed continuous variables were assessed using independent-samples $t$-test and those with respect to non-normally distributed variables were assessed using MannWhitney $U$-test. Categorical variables were presented as frequency (proportions) and compared using Chi-squared test. Multivariate logistic regression was performed to further test the strength of association of sarcopenia with ICW and MAMC, after controlling for potential confounding variables including age, sex, FM, VFA, WBC, HB, PLT, UA, LDH. All statistical analyses were performed using SPSS 25 (IBM, Armonk, New York, NY, USA). Two-sided $P$ values $<0.05$ were considered indicative of statistical significance for all comparisons.

\section{Results}

\section{Changes in Clinical Indicators After Chemotherapy}

Eighty-seven patients with AML (non-M3) at initial diagnosis were eligible for inclusion. Of these, 69 (79.3\%) participants achieved CR and subsequently accepted both BIA and three consolidation chemotherapy sessions. Table 1 shows the comparison of pre- and post-chemotherapy clinical and laboratory data. Post-chemotherapy BMI, white blood cell count (WBC), uric acid (UA), serum albumin (ALB), and lactate dehydrogenase (LDH) levels were significantly lower than the corresponding baseline levels $(P<0.05$ for all), while post-chemotherapy platelet count (PLT) was significantly higher than the baseline PLT (median 117 vs 51, $P<0.001$ ). There was no significant difference between the two groups with respect to other indices, including systolic blood pressure, diastolic blood pressure, or hemoglobin (HB). 
Table I Changes in Clinical Indicators After Chemotherapy

\begin{tabular}{|c|c|c|c|}
\hline \multirow[t]{2}{*}{ Variables } & \multicolumn{2}{|c|}{ AML (Non-M3) } & \multirow[t]{2}{*}{$P$ value } \\
\hline & $\begin{array}{l}\text { Pre-Chemotherapy }(n=69): \\
\text { Initial Diagnosis }\end{array}$ & $\begin{array}{l}\text { Post-Chemotherapy ( } n=69): \text { Three } \\
\text { Chemotherapy Sessions }\end{array}$ & \\
\hline \multicolumn{4}{|l|}{ Clinical data } \\
\hline Sex (male) & $37(53.6)$ & I & I \\
\hline Age & $53(44.5-61.0)$ & l & I \\
\hline BMI $\left(\mathrm{kg} / \mathrm{m}^{2}\right)$ & $22.1(20.3-24.6)$ & $20.8(19.2-23.7)$ & 0.048 \\
\hline $\mathrm{SBP}(\mathrm{mmHg})$ & $120.7 \pm \mid 2.7$ & $122.5 \pm 15.9$ & 0.280 \\
\hline $\mathrm{DBP}(\mathrm{mmHg})$ & $70.7 \pm 9.9$ & $74.1 \pm 14.9$ & 0.307 \\
\hline \multicolumn{4}{|l|}{ Laboratory data } \\
\hline WBC $\left(10^{9} / \mathrm{L}\right)$ & $11.0(2.9-39.6)$ & $4.2(2.4-8.2)$ & 0.011 \\
\hline $\operatorname{RBC}\left(10^{12} / \mathrm{L}\right)$ & $2.2(1.7-3.1)$ & $2.6(2.1-2.8)$ & 0.034 \\
\hline N\% & $17.6(3.0-42.1)$ & $55.4(32.4-68.9)$ & $<0.001$ \\
\hline $\mathrm{HB}(\mathrm{g} / \mathrm{L})$ & $69.8(56-94)$ & $76(67.6-86.8)$ & 0.150 \\
\hline PLT $\left(10^{9} / \mathrm{L}\right)$ & $51(22-139)$ & II $7.0(57.3-2 \mid 5.5)$ & $<0.001$ \\
\hline UA (mmol/L) & $318.7 \pm 106.1$ & $242.7 \pm 119.5$ & $<0.001$ \\
\hline ALB $(g / L)$ & $40(38-43)$ & $35.5(33.0-38.0)$ & $<0.001$ \\
\hline LDH (U/L) & $584(26|-| 403)$ & 16I (114-264) & $<0.001$ \\
\hline \multicolumn{4}{|c|}{ Cytogenetic risk group } \\
\hline Favorable & $31(45)$ & I & l \\
\hline Intermediate & $14(20.3)$ & I & I \\
\hline Adverse & $24(34.7)$ & I & I \\
\hline
\end{tabular}

Abbreviations: BMI, body mass index; SBP, systolic blood pressure; DBP, diastolic blood pressure; WBC, white blood cell; RBC, red blood cell; N\%, neutrophil\%; HB, hemoglobin; PLT, platelet; UA, uric acid; ALB, albumin; LDH, lactate dehydrogenase.

\section{Changes in Body Composition Indices After Chemotherapy}

The BIA parameters are shown in Table 2. Post-chemotherapy ASM and HGS levels were significantly lower than those before chemotherapy. The prevalence of sarcopenia in our cohort after chemotherapy was significantly higher than that before chemotherapy $(39.1 \%$ vs $14.5 \%, P<0.001)$. After chemotherapy, there was a significant decrease in the three muscle indices (ie, SMM, FFM, and SLM). In addition, the value of MAMC, ICW, and TBW significantly decreased after chemotherapy $(P<0.05)$. There was no significant effect of chemotherapy on FM or VFA $(P>0.05)$.

\section{Correlation Between Sarcopenia and BIA Indices}

Correlation between sarcopenia and BIA indices was assessed by Spearman correlation analysis (Table 3). BIA indices including ASM $(r=0.889, P<0.001)$, ICW $(r=0.869, P<0.001)$, and MAMC $(r=0.849, P<0.001)$ showed a positive correlation with SMI. ASM $(r=-0.453 P=0.001)$, ICW $(r=-0.322, P<0.05)$, MAMC $(r=-0.352, P<0.05)$ showed a negative correlation with sarcopenia. VFA and FM showed no correlation with SMI or sarcopenia $(P>0.05)$. 
Table 2 Pre- and Post-Chemotherapy Bioelectric Impedance Analysis Parameters

\begin{tabular}{|c|c|c|c|}
\hline \multirow[t]{2}{*}{ Variables } & \multicolumn{2}{|c|}{ AML (Non-M3) } & \multirow[t]{2}{*}{$P$ value } \\
\hline & $\begin{array}{l}\text { Pre-Chemotherapy }(n=69): \\
\text { Initial Diagnosis }\end{array}$ & $\begin{array}{l}\text { Post-Chemotherapy ( } n=69): \text { Three } \\
\text { Chemotherapy Sessions }\end{array}$ & \\
\hline \multicolumn{4}{|c|}{ Diagnosis of sarcopenia } \\
\hline ASM (kg) & $18.0(|5.2-2| .0)$ & $16.3(14.4-19.6)$ & 0.053 \\
\hline HGS (kg) & $30.6 \pm 11.7$ & $24.6 \pm 10.9$ & 0.003 \\
\hline $\mathrm{SMI}\left(\mathrm{kg} / \mathrm{m}^{2}\right)$ & $7.1(6.15-7.7)$ & $6.5(5.7-7.2)$ & 0.007 \\
\hline Sacropenia $(\mathrm{n}, \%)$ & $10(14.5)$ & $27(39.1)$ & $<0.001$ \\
\hline \multicolumn{4}{|l|}{ BIA data } \\
\hline SMM (kg) & $24.1(21.3-27.2)$ & $21.6(20.2-24.5)$ & 0.010 \\
\hline FFM (kg) & $44.2(39.7-49.4)$ & $40.3(37.9-46.0)$ & 0.022 \\
\hline SLM (kg) & $41.8(35.4-46.8)$ & $37.9(35.7-43.2)$ & 0.017 \\
\hline ICW (\%) & $20.0(17.6-22.4)$ & I8.I (I7.0-20.3) & 0.010 \\
\hline TBW (\%) & $32.7(29.1-36.6)$ & $29.6(27.9-34.0)$ & 0.020 \\
\hline $\operatorname{MAMC}(\mathrm{cm})$ & $23.6(21.9-24.8)$ & $22.1(21.0-23.5)$ & 0.003 \\
\hline VFA $\left(\mathrm{cm}^{2}\right)$ & $55.8(4 I .8-75 . I)$ & $64.0(50.0-79.6)$ & 0.169 \\
\hline $\mathrm{FM}(\mathrm{kg})$ & $13.1(9.6-16.4)$ & $12.6(10.0-17.2)$ & 0.822 \\
\hline
\end{tabular}

Abbreviations: ASM, appendicular skeletal muscle mass; HGS, hand grip strength; SMI, appendicular skeletal mass index which is defined as ASM/height ${ }^{2}$; SMM, skeletal muscle mass; FFM, fat-free mass; SLM, soft lean mass; ICW, intracellular water; TBW, total body water; MAMC, mid-arm muscle circumference; VFA, visceral fat area; FM, fat mass.

Table 3 Correlation Coefficients (Spearman's Rho) for Sarcopenia and BIA Indicators

\begin{tabular}{|l|c|c|c|c|}
\hline \multirow{2}{*}{ Variables } & \multicolumn{2}{|c|}{ SMI } & \multicolumn{2}{c|}{ Sarcopenia } \\
\cline { 2 - 5 } & $\boldsymbol{r}$ & $\boldsymbol{P}$ value & $\boldsymbol{r}$ & $\boldsymbol{P}$ value \\
\hline ASM $(\mathrm{kg})$ & 0.889 & $<0.001$ & -0.453 & 0.001 \\
\hline ICW $(\%)$ & 0.869 & $<0.001$ & -0.322 & 0.029 \\
\hline MAMC (cm) & 0.849 & $<0.001$ & -0.352 & 0.015 \\
\hline VFA (cm $\left.{ }^{2}\right)$ & 0.169 & 0.257 & -0.192 & 0.195 \\
\hline FM $(\mathrm{kg})$ & 0.142 & 0.342 & -0.170 & 0.254 \\
\hline
\end{tabular}

Abbreviations: ASM, appendicular skeletal muscle mass; ICW, intracellular water; MAMC, mid-arm muscle circumference; VFA, visceral fat area; FM, fat mass.

\section{Logistic Regression Analysis of the Association of Sacropenia with MAMC and ICW}

Logistic regression analysis was performed to evaluate the strength of the association of sarcopenia with ICW and MAMC (Table 4). In model 2, after adjusting for age, sex, VFA, FM, WBC, HB, PLT, UA, and LDH, increased ICW showed a significant association with decreased sarcopenia [odds ratio (OR): 0.50 ; 95\% confidence interval (CI) 0.30 0.82]. Each additional unit of MAMC was associated with $71 \%$ lower risk of sarcopenia (OR: 0.29 ; $95 \%$ CI $0.13-0.66$ ) in AML (non-M3) patients after chemotherapy. 
Table 4 Results of Logistic Regression Analysis Showing the Association of BMI, AMC, and ICW with the Incidence of Sarcopenia

\begin{tabular}{|l|c|c|c|}
\hline \multirow{3}{*}{ Variables } & \multicolumn{3}{|c|}{ Sarcopenia (n=27) } \\
\cline { 2 - 4 } & Model & OR (95\% CI) & P value \\
\hline \multirow{3}{*}{ ICW } & Crude OR & $0.84(0.73-0.97)$ & 0.023 \\
\cline { 2 - 4 } & Model I & $0.67(0.5 I-0.89)$ & 0.007 \\
\cline { 2 - 4 } & Model 2 & $0.50(0.30-0.82)$ & 0.007 \\
\hline \multirow{3}{*}{ MAMC } & Crude OR & $0.67(0.48-0.92)$ & 0.015 \\
\cline { 2 - 4 } & Model I & $0.42(0.23-0.75)$ & 0.004 \\
\cline { 2 - 4 } & Model 2 & $0.29(0.13-0.66)$ & 0.003 \\
\hline
\end{tabular}

Notes: Model I: adjusted for age, sex; Model 2: adjusted for age, sex, FM, VFA, WBC, HB, PLT, UA, LDH.

Abbreviations: ICW, intracellular water; MAMC, mid-arm muscle circumference.

\section{Discussion}

The key findings of our study are as follows: a) In AML patients, the increased incidence of sarcopenia after chemotherapy depends on weight reduction, which is mainly due to the loss of muscle mass and water content rather than fat mass. b) MAMC and ICW showed a significant association with the incidence of sarcopenia after adjusting for age, sex, and clinical indicators.

Sarcopenia caused by changes in body composition is often associated with poor prognosis in cancer patients. Sarcopenia is characterized by loss of skeletal muscle mass and performance and is a risk factor for frailty, morbidity, and mortality in older people. ${ }^{14,15}$ Moreover, low skeletal muscle has been shown to be a prognostic marker in the context of many diseases such as malignant cancer, heart failure, and chronic obstructive pulmonary disease. ${ }^{16-18}$ Several studies have shown that body composition, as assessed by CT, is useful in predicting the prognosis; in addition, CT-detected sarcopenia was associated with lower overall survival in patients with AML and acute lymphoblastic leukemia (ALL). ${ }^{19,20}$

Our study revealed changes in body composition and increased prevalence of sarcopenia after chemotherapy. There was a simultaneous decline in BMI, muscle and water content without changes in fat content, although the biochemical parameters (WBC, PLT, ALB and LDH) improved after three chemotherapy sessions. Myelosuppression is a common side effect of anthracyclines. For prevention of complications such as bleeding and infection, patients are often advised bed rest. ${ }^{21}$ Previous studies have shown that bed rest substantially reduces skeletal muscle mass without affecting lipid content and is associated with changes in metabolic phenotype including decline in resting fat oxidation, basal metabolic rate, ${ }^{22}$ glucose tolerance and insulin sensitivity, ${ }^{23}$ and increase in mitochondrial production of reactive oxygen species (ROS). ${ }^{24}$ Therefore, treatment regimens of sarcopenia may be for individual and selected cases, including physical training, modifications of nutritional intake, and pharmacological treatment. ${ }^{25}$

There is a distinct difference between body composition disorder induced by chemotherapy and cachexia. Cancer cachexia is related to poor prognosis and is defined as atrophy of skeletal muscle and adipose tissue resulting in progressive weight loss. ${ }^{26}$ It has been frequently related to involuntary weight loss, decreased muscle mass as well as biochemical changes, such as decreased ALB. ${ }^{27}$ Of note, the post-chemotherapy weight loss in our cohort was mainly due to loss of muscle mass and water instead of fat. However, the underlying mechanism of this phenomenon is not clear. Several reasons may explain this phenomenon. Firstly, both adipocytes and fat tissue affect metabolism and inactivation of anthracyclines. Secondly, this phenomenon may be related to the inflammatory response of AML with sarcopenia. A substantial body of literature suggests that inflammatory cytokines activate many molecular pathways in skeletal muscle rather than adipose tissue, ${ }^{28}$ and high level of inflammatory cytokines was shown to negatively correlate with skeletal muscle mass. ${ }^{29}$

BIA is a simple, relatively cheap, and effective approach to evaluate body composition. High BMI is associated with increased risk of most hematological malignancies. ${ }^{30}$ Although BMI is most frequently used to assess nutritional status, it 
does not precisely reflect body composition, nor does it differentiate skeletal muscle from body fat. ${ }^{31}$ Owing to its advantages, BIA has been widely used for body composition analysis in research of various diseases over the past few decades. ${ }^{11,32}$ Many BIA indicators including segmental skeletal muscle, body fat and water content provide useful supportive information to facilitate clinical diagnosis. ${ }^{33,34}$ Here, we measured body composition indicators including skeletal muscle mass using BIA with the aim of more accurate characterization of the link between sarcopenia and AML.

MAMC measured by BIA can reflect muscle mass and performance and has been used as an indicator of nutritional status. ${ }^{35}$ Many previous studies have demonstrated a correlation between MAMC and skeletal muscle index, which has been proven valuable to assess the outcomes in patients with hematological malignancies. ${ }^{36,37}$ Due to better predictable effects of reflecting muscle performance, intracellular water (ICW) may be used as a supplementary indicator together with MAMC for the diagnosis of sarcopenia. ${ }^{38,39}$ In the present study, both MAMC and ICW measured by BIA showed a strong correlation with post-chemotherapy sarcopenia in AML patients, which is consistent with previous studies. This association persisted after adjustment for a variety of potential confounding factors.

Some limitations of our study should be acknowledged. Firstly, this was a single center cross-sectional study with a small sample size. Secondly, we used BIA to measure body composition instead of other modalities such as dual-energy X-ray absorptiometry (DEXA), computed tomography (CT), and magnetic resonance imaging (MRI); the accuracy of BIA is still controversial. Thirdly, we only observed the incidence of sarcopenia based on changes in BIA indicators. We were unable to call the patients back for a comprehensive assessment of sarcopenia.

\section{Conclusion}

We found increased incidence of sarcopenia in AML (non-M3) patients after chemotherapy. Both MAMC and ICW measured by BIA showed a strong association with sarcopenia. Our findings further our understanding of sarcopenia in AML patients.

\section{Acknowledgment}

This study was funded by grants from Chongqing Medical Research Project: Study on early cardiotoxicity of antitumor drugs in lymphoma patients (Grant No. 2021MSXM276); and Chongqing Natural Science Foundation general project: the mechanism of doxorubicin promoting atherosclerosis in lymphoma patients through NF- $\mathrm{KB} / \mathrm{miR}-33$ signaling pathway (Grant No. cstc2019jcyj-msxmX0043).

\section{Disclosure}

The authors report no conflicts of interest in this work.

\section{References}

1. Döhner H, Weisdorf DJ, Bloomfield CD. Acute myeloid leukemia. N Engl J Med. 2015;373(12):1136-1152. doi:10.1056/NEJMra1406184

2. De kouchkovsky I, Abdul-Hay M. Acute myeloid leukemia: a comprehensive review and 2016 update. Blood Cancer J. 2016;6(7):e441-e441. doi:10.1038/bcj.2016.50

3. Kadia TM, Ravandi F, Cortes J, Kantarjian H. New drugs in acute myeloid leukemia. Ann Oncol. 2016;27(5):770-778. doi:10.1093/annonc/ mdw015

4. Bauer J, Morley JE, Schols AMWJ, et al. Sarcopenia: a time for action. An SCWD position paper. J Cachexia Sarcopenia Muscle. 2019;10 (5):956-961. doi: $10.1002 /$ jcsm. 12483

5. Kamiya T, Mizuno K, Ogura S, et al. A prospective observational study evaluating sarcopenia by using the bioelectrical impedance analysis in elderly patients with hematologic malignancies. Blood. 2018;132(Supplement 1):4851. doi:10.1182/blood-2018-99-114545

6. Martin L, Senesse P, Gioulbasanis I, et al. Diagnostic criteria for the classification of cancer-associated weight loss. J Clin Oncol. 2015;33 (1):90-99. doi:10.1200/JCO.2014.56.1894

7. Dewys WD, Begg C, Lavin PT, et al. Prognostic effect of weight loss prior to chemotherapy in cancer patients. Am J Med. 1980;69(4):491-497. doi:10.1016/S0149-2918(05)80001-3

8. van Lieshout R, Tick LW, Dieleman JP, et al. Changes in body weight and serum liver tests associated with parenteral nutrition compared with no parenteral nutrition in patients with acute myeloid leukemia during remission induction treatment. Support Care Cancer. 2020;28(9):4381-4393. doi:10.1007/s00520-019-05251-9

9. Sun Q, Ming H, Qian S, Li JY. Sarcopenia as a significant prognostic factor in acute myeloid leukemia: validation of a novel scoring system. Blood. 2019;134(Supplement_1):5896. doi:10.1182/blood-2019-130517

10. Kyle UG, Bosaeus I, De Lorenzo AD, et al. Bioelectrical impedance analysis-part II: utilization in clinical practice. Clin Nutr. 2004;23 (6):1430-1453. doi:10.1016/j.clnu.2004.09.012 
11. Ward LC. Bioelectrical impedance analysis for body composition assessment: reflections on accuracy, clinical utility, and standardisation. Eur J Clin Nutr. 2019;73(2):194-199. doi:10.1038/s41430-018-0335-3

12. Mathiowetz V, Kashman N, Volland G, Weber K, Dowe M, Rogers S. Grip and pinch strength: normative data for adults. Arch Phys Med Rehabil. 1985;66(2):69-74.

13. Chen LK, Woo J, Assantachai P, et al. Asian working group for sarcopenia: 2019 consensus update on sarcopenia diagnosis and treatment. $J A m$ Med Dir Assoc. 2020;21(3):300-307.e2. doi:10.1016/j.jamda.2019.12.012

14. Pin F, Barreto R, Couch ME, Bonetto A, O'Connell TM. Cachexia induced by cancer and chemotherapy yield distinct perturbations to energy metabolism. J Cachexia Sarcopenia Muscle. 2019;10(1):140-154. doi:10.1002/jcsm.12360

15. Argilés JM, Busquets S, Stemmler B, López-Soriano FJ. Cancer cachexia: understanding the molecular basis. Nat Rev Cancer. 2014;14 (11):754-762. doi:10.1038/nrc3829

16. Hopkins JJ, Reif RL, Bigam DL, Baracos VE, Eurich DT, Sawyer MB. The impact of muscle and adipose tissue on long-term survival in patients with stage I to III colorectal cancer. Dis Colon Rectum. 2019;62(5):549-560. doi:10.1097/DCR.0000000000001352

17. Huang BT, Peng Y, Liu W, et al. Lean mass index, body fat and survival in Chinese patients with coronary artery disease. QJM. 2015;108 (8):641-647. doi:10.1093/qjmed/hcv013

18. Kou HW, Yeh CH, Tsai HI, et al. Sarcopenia is an effective predictor of difficult-to-wean and mortality among critically ill surgical patients. PLoS One. 2019;14(8):e0220699. doi:10.1371/journal.pone.0220699

19. Surov A, Wienke A. Sarcopenia predicts overall survival in patients with malignant hematological diseases: a meta-analysis. Clin Nutr. 2021;40 (3):1155-1160. doi:10.1016/j.clnu.2020.07.023

20. Jung J, Lee E, Shim H, Park JH, Eom HS, Lee H. Prediction of clinical outcomes through assessment of sarcopenia and adipopenia using computed tomography in adult patients with acute myeloid leukemia. Int J Hematol. 2021;114(1):44-52. doi:10.1007/s12185-021-03122-w

21. Alibhai SMH, O'Neill S, Fisher-Schlombs K, et al. A clinical trial of supervised exercise for adult inpatients with acute myeloid leukemia (AML) undergoing induction chemotherapy. Leuk Res. 2012;36(10):1255-1261. doi:10.1016/j.leukres.2012.05.016

22. Bergouignan A, Schoeller DA, Normand S, et al. Effect of physical inactivity on the oxidation of saturated and monounsaturated dietary fatty acids: results of a randomized trial. PLOS Clin Trial. 2006;1(5):e27. doi:10.1371/journal.pctr.0010027

23. Dirks ML, Wall BT, van de Valk B, et al. One week of bed rest leads to substantial muscle atrophy and induces whole-body insulin resistance in the absence of skeletal muscle lipid accumulation. Diabetes. 2016;65(10):2862-2875. doi:10.2337/db15-1661

24. Gram M, Vigelsø A, Yokota T, Helge JW, Dela F, Hey-Mogensen M. Skeletal muscle mitochondrial $\mathrm{H}_{2} \mathrm{O}_{2}$ emission increases with immobilization and decreases after aerobic training in young and older men: impaired human mitochondrial function after immobilization. $J$ Physiol. 2015;593(17):4011-4027. doi:10.1113/JP270211

25. Denison HJ, Cooper C, Sayer AA, Robinson SM. Prevention and optimal management of sarcopenia: a review of combined exercise and nutrition interventions to improve muscle outcomes in older people. Clin Interv Aging. 2015;10:859-869. doi:10.2147/CIA.S55842

26. Fearon K, Strasser F, Anker SD, et al. Definition and classification of cancer cachexia: an international consensus. Lancet Oncol. 2011;12 (5):489-495. doi:10.1016/S1470-2045(10)70218-7

27. Fearon KC, Voss AC, Hustead DS. Definition of cancer cachexia: effect of weight loss, reduced food intake, and systemic inflammation on functional status and prognosis. Am J Clin Nutr. 2006;83(6):1345-1350. doi:10.1093/ajen/83.6.1345

28. Edward J, Sang-Rok L, Bong-Sup P, Jeong-Su K. Potential mechanisms underlying the role of chronic inflammation in age-related muscle wasting. Aging Clin Exp Res. 2012;24:5. doi:10.3275/8464

29. Ito S, Nakashima H, Ando K, et al. Association between low muscle mass and inflammatory cytokines. Biomed Res Int. 2021;2021:1-7. doi: $10.1155 / 2021 / 5572742$

30. De Ridder J, Julián-Almárcegui C, Mullee A, et al. Comparison of anthropometric measurements of adiposity in relation to cancer risk: a systematic review of prospective studies. Cancer Causes Control. 2016;27(3):291-300. doi:10.1007/s10552-015-0709-y

31. Sarkar SR, Kuhlmann MK, Kotanko P, et al. Metabolic consequences of body size and body composition in hemodialysis patients. Kidney Int. 2006;70(10):1832-1839. doi:10.1038/sj.ki.5001895

32. Kilic MK, Kizilarslanoglu MC, Arik G, et al. Association of bioelectrical impedance analysis-derived phase angle and sarcopenia in older adults. Nutr Clin Pract. 2017;32(1):103-109. doi:10.1177/0884533616664503

33. Irisawa H, Mizushima T. Correlation of body composition and nutritional status with functional recovery in stroke rehabilitation patients. Nutrients. 2020;12(7):1923. doi:10.3390/nu12071923

34. Chen J, Lu K, Chen H, et al. Trunk skeletal muscle mass and phase angle measured by bioelectrical impedance analysis are associated with the chance of femoral neck fracture in very elderly people. CIA. 2020;15:889-895. doi:10.2147/CIA.S250629

35. Landi F, Russo A, Liperoti R, et al. Midarm muscle circumference, physical performance and mortality: results from the aging and longevity study in the Sirente geographic area (ilSIRENTE study). Clin Nutr. 2010;29(4):441-447. doi:10.1016/j.clnu.2009.12.006

36. Chang PK, Chen WL, Wu LW. Mid-arm muscle circumference: a significant factor of all-cause and cancer mortalities in individuals with elevated platelet-to-lymphocyte ratio. PLoS One. 2018;13(12):e0208750. doi:10.1371/journal.pone.0208750

37. Noori N, Kopple JD, Kovesdy CP, et al. Mid-arm muscle circumference and quality of life and survival in maintenance hemodialysis patients. CJASN. 2010;5(12):2258-2268. doi:10.2215/CJN.02080310

38. Serra-Prat M, Lorenzo I, Palomera E, Yébenes J, Campins L, Cabré M. Intracellular water content in lean mass is associated with muscle strength, functional capacity, and frailty in community-dwelling elderly individuals. A cross-sectional study. Nutrients. 2019;11(3):661. doi:10.3390/ nu11030661

39. Hetherington-Rauth M, Baptista F, Sardinha LB. BIA-assessed cellular hydration and muscle performance in youth, adults, and older adults. Clin Nutr. 2020;39(8):2624-2630. doi:10.1016/j.clnu.2019.11.040 


\section{Publish your work in this journal}

The International Journal of General Medicine is an international, peer-reviewed open-access journal that focuses on general and internal medicine, pathogenesis, epidemiology, diagnosis, monitoring and treatment protocols. The journal is characterized by the rapid reporting of reviews, original research and clinical studies across all disease areas. The manuscript management system is completely online and includes a very quick and fair peer-review system, which is all easy to use. Visit http://www.dovepress.com/testimonials.php to read real quotes from published authors.

Submit your manuscript here: https://www.dovepress.com/international-journal-of-general-medicine-journal 